\title{
OPEN Survival Genie, a web platform for survival analysis across pediatric and adult cancers
}

\author{
Bhakti Dwivedii ${ }^{1}$, Hope Mumme ${ }^{4}$, Sarthak Satpathy ${ }^{4}$, Swati S. Bhasin ${ }^{2,3}$ \& \\ Manoj Bhasin ${ }^{1,2,3,4,5 凶}$
}

The genomics data-driven identification of gene signatures and pathways has been routinely explored for predicting cancer survival and making decisions related to targeted treatments. A large number of packages and tools have been developed to correlate gene expression/mutations to the clinical outcome but lack the ability to perform such analysis based on pathways, gene sets, and gene ratios. Furthermore, in this single-cell omics era, the cluster markers from cancer single-cell transcriptomics studies remain an underutilized prognostic option. Additionally, no bioinformatics online tool evaluates the associations between the enrichment of canonical cell types and survival across cancers. Here we have developed Survival Genie, a web tool to perform survival analysis on single-cell RNAseq (scRNA-seq) data and a variety of other molecular inputs such as gene sets, genes ratio, tumorinfiltrating immune cells proportion, gene expression profile scores, and tumor mutation burden. For a comprehensive analysis, Survival Genie contains 53 datasets of 27 distinct malignancies from 11 different cancer programs related to adult and pediatric cancers. Users can upload scRNA-seq data or gene sets and select a gene expression partitioning method (i.e., mean, median, quartile, cutp) to determine the effect of expression levels on survival outcomes. The tool provides comprehensive results including box plots of low and high-risk groups, Kaplan-Meier plots with univariate Cox proportional hazards model, and correlation of immune cell enrichment and molecular profile. The analytical options and comprehensive collection of cancer datasets make Survival Genie a unique resource to correlate gene sets, pathways, cellular enrichment, and single-cell signatures to clinical outcomes to assist in developing next-generation prognostic and therapeutic biomarkers. Survival Genie is open-source and available online at https://bbisr.shinyapps.winship.emory.edu/SurvivalGe nie/.

Over the past decade advancement in genomics and epigenomics technologies has produced a large amount of data to understand the complex mechanisms of cancer. This accessibility and affordability of genome sequencing resulted in the completion of multiple ambitious projects in the cancer arena such as The Cancer Genome Atlas $(\mathrm{TCGA})^{1}$, the International Cancer Genome Consortium (ICGC) ${ }^{2}$, and the Therapeutically Applicable Research to Generate Effective Treatments (TARGET) ${ }^{3}$ to generate a comprehensive molecular landscape of different cancers. TCGA has profiled genetic and epigenetics landscapes of $\sim 20,000$ tumor samples across 33 major adult cancer types to generate novel insights for better diagnosis and treatment of cancer. Similarly, the TARGET ${ }^{3}$ program has generated comprehensive genetics, epigenetics, and transcriptome profiles of 6000 samples from nine pediatrics tumor types to understand molecular mechanisms and develop novel therapies. These ambitious projects provided access to omics, clinical, demographic, and outcome information through the Genomic Data Commons (GDC) gateway ${ }^{4}$ to understand the impact of various associated factors on cancer outcomes besides collecting molecular information. The correlative analysis of these vast omics and clinical data can assist in identifying robust prognostic biomarkers to predict cancer outcomes. However, given the inherent complexity of data coupled with the sheer volume from numerous initiatives, the overall potential is largely reduced due to the lack of systematic and user-friendly online analytical tools. Utilization by a larger number of users will

\footnotetext{
${ }^{1}$ Winship Cancer Institute, Emory University, Atlanta, GA, USA. ${ }^{2}$ Aflac Cancer and Blood Disorders Center, Children's Healthcare of Atlanta, Atlanta, GA, USA. ${ }^{3}$ Department of Pediatrics, Emory University, Atlanta, GA, USA. ${ }^{4}$ Department of Biomedical Informatics, Emory University, Atlanta, GA, USA. ${ }^{5}$ Aflac Cancer and Blood Disorders Center, Emory School of Medicine, Children Healthcare of Atlanta, Woodruff Memorial Research Building, Room 4107, 101 Woodruff Circle, 4th Floor East, Atlanta, GA 30322, USA. ${ }^{\varpi}$ email: manoj.bhasin@ emory.edu
} 
require data access without the need to download or manipulate files in programming languages such as Python and $\mathrm{R}$. This raises the need for the development of user-friendly online platforms for the exploration of this data.

To address this unmet need, multiple online platforms for exploring the association of genes and proteins with the clinical outcome have been developed ${ }^{5}$. Among the most used survival analysis tools are the $\mathrm{cBioPortal}^{6}$ and GDC data portal ${ }^{4}$, providing users exploratory analyses of multi-omics cancer datasets and survival analysis of single gene-based on DNA alterations only. UALCAN ${ }^{7}$ is an interactive web resource for analyzing cancer OMICS data from TCGA, MET500, and CPTAC to correlate gene, protein, and miRNA expression profile and patient survival information. TRGAted ${ }^{8}$ tool allows survival analysis of single and multiple proteins across TCGA cancer types. This tool has clinical options with multiple optimal cut-offs, but it is limited to protein data and uses the mean expression as a cut-point for querying multiple proteins. Likewise, the KMplotter ${ }^{9,10}$ allows studying the clinical impact of individual genes in cancers from TCGA, GEO, and EGA using different splitting methods (e.g., median, percentile, quartiles). In this tool, users can upload multiple genes, ratios of two genes, and mean or median expression of genes, yet analysis will be performed at the individual gene level. The tool also allows restrictive analysis based on clinical subtypes and the proportion of immune cells. In this series, LOGpc ${ }^{11}$ is another web server that estimates survival in 27 cancer types from TCGA and GEO using RNA-seq data but limited to single gene-based analysis. A recently developed tool, ESurv ${ }^{12}$ also provides univariate survival analysis by a single gene or cancer type from TCGA datasets.

Multiple genes, gene sets, and canonical pathways play a key role in defining the different subtypes of cancers with varying aggressiveness and outcomes. This raises the need for a tool to perform survival analysis from a set of genes rather than on a single gene basis within a set. Keeping this in mind, we developed the Survival Genie platform by compiling a comprehensive list of adult and pediatric cancers along with the implementation of multiple approaches for prognostic genomics features identification. For comprehensive survival evaluation, the Survival Genie contains 53 datasets of 27 distinct malignancies from 11 different cancer programs for both adult and pediatric cancers. Users can upload single-cell data or gene sets and select partitioning methods (i.e., mean, median, quartile, and cutp) to determine the effect of expression levels on survival outcomes. The tool provides comprehensive results including box plots of low and high-risk groups, Kaplan-Meier plots with univariate Cox proportional hazards model, and correlation of immune cell enrichment and molecular profile.

\section{Methods}

Collection and processing of cancer data. Survival Genie contains 53 datasets of 27 distinct malignancies from 11 different cancer programs for both adult and pediatric cancers (Supplementary Table 1). All the clinical and genomics (transcriptomic and mutation) data were downloaded from the GDC data portal ${ }^{4}$ using the GenomicDataCommons Bioconductor R package ${ }^{13}$. The samples with paired survival (e.g., days to followup, days to death, vital status), mRNA-seq, and whole exome sequencing are only used for survival analysis. The three tumor types (primary, recurrent, and metastatic) are included in the analysis depending on the cancer type. Additionally, for correlating the survival information with immune infiltrate, the clinical Histology and Eosin (H\&E) digital image-based quantification of tumor-infiltrating lymphocytes (TILs) were obtained from the Cancer Imaging Archive ${ }^{14}$ for the 13 TCGA datasets.

Survival analysis. Survival Genie performs overall survival (OS) and event-free survival (EFS) statistical analysis as implemented in the 'survival' $\mathrm{R}$ package ${ }^{15}$. Kaplan-Meier survival curves are used to estimate the OS/ EFS using the survfit function and a log-rank test is done to compute differences in OS/EFS between the defined high- and low-risk groups. Univariate analysis with Cox proportional hazards regression model is performed on the patient data using coxph function in R/Bioconductor ${ }^{16}$. The survival association is considered significant if the $p$-values for the log-rank and Wald tests are lower than 0.05 .

Implementation. The Survival Genie source code is written in the R programming language ${ }^{17}$ and the interactive web-server is implemented using the shiny $\mathrm{R}$ package ${ }^{18}$. Cancer clinical and genomics datasets for survival analyses are processed and analyzed using Perl and $\mathrm{R}$ languages. The tool has been extensively tested on multiple operating systems (Linux, Mac, Windows) and web-browsers (Chrome, Firefox, and Safari). The tool is currently hosted on a 64-bit CentOS 6 backend server running the Shiny Server program designed to host $\mathrm{R}$ Shiny applications ${ }^{18}$. The tool along with source code is also available on the GitHub repository https://github. $\mathrm{com} / \mathrm{bhasin}-\mathrm{lab} /$ SurvivalGenie. A detailed step-wise analysis tutorial about the tool is also available on YouTube at https://www.youtube.com/watch?v=H5s6OYvwwoo.

\section{Results}

Development of Survival Genie. Figure 1 shows the overview of the user-friendly Survival Genie web interface for choosing analysis type, inputs, cancer datasets, parameters to explore and visualize the effect of dichotomous molecular (e.g., gene) profiles on patient survival outcomes. Detailed documentation for input and output parameters, and example data are available on the help page of the Survival Genie web server.

Pathways, gene sets, and systems biology modules-based survival analysis. Survival Genie provides a range of input options (i.e., gene-based, cell-based, profile-based, and mutation-based) to allow flexible and comprehensive survival analysis based on the user's needs (Fig. 2, steps 1-2). (a) Gene-based input option requires HGNC approved gene symbols as input, the most popular and widely used option for identifying prognostic gene markers. Gene Sets allow the input of a list of genes from pathways or biological processes as a gene set. An aggregated enrichment score is computed for a gene set using the single-sample gene set enrichment method (ssGSEA ${ }^{19}$ ) to estimate optimal cut-off in predicting survival outcomes. The gene set option also allows submission of a 


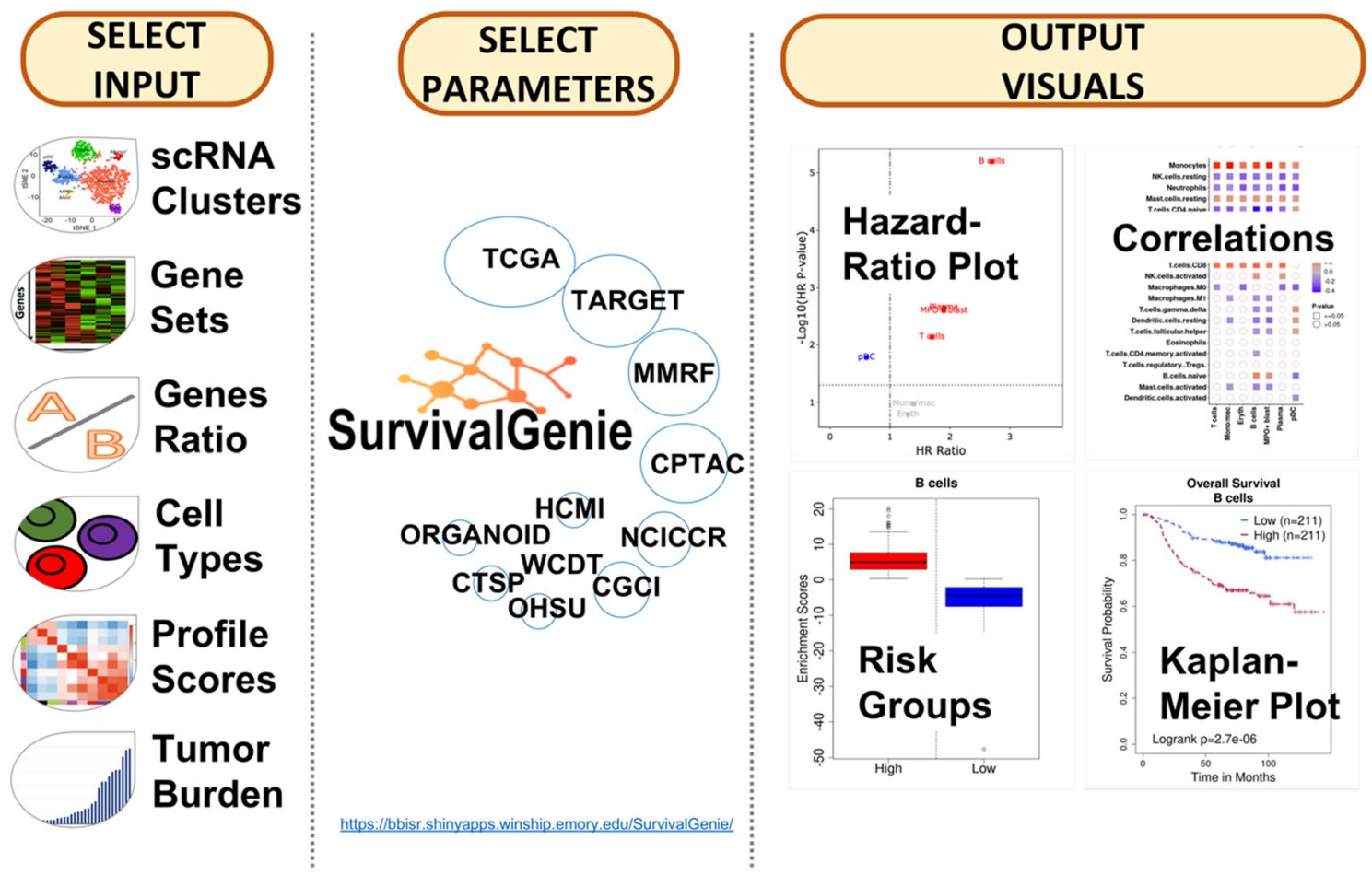

Figure 1. An overview of the Survival Genie platform showing input, analysis parameters, and output.

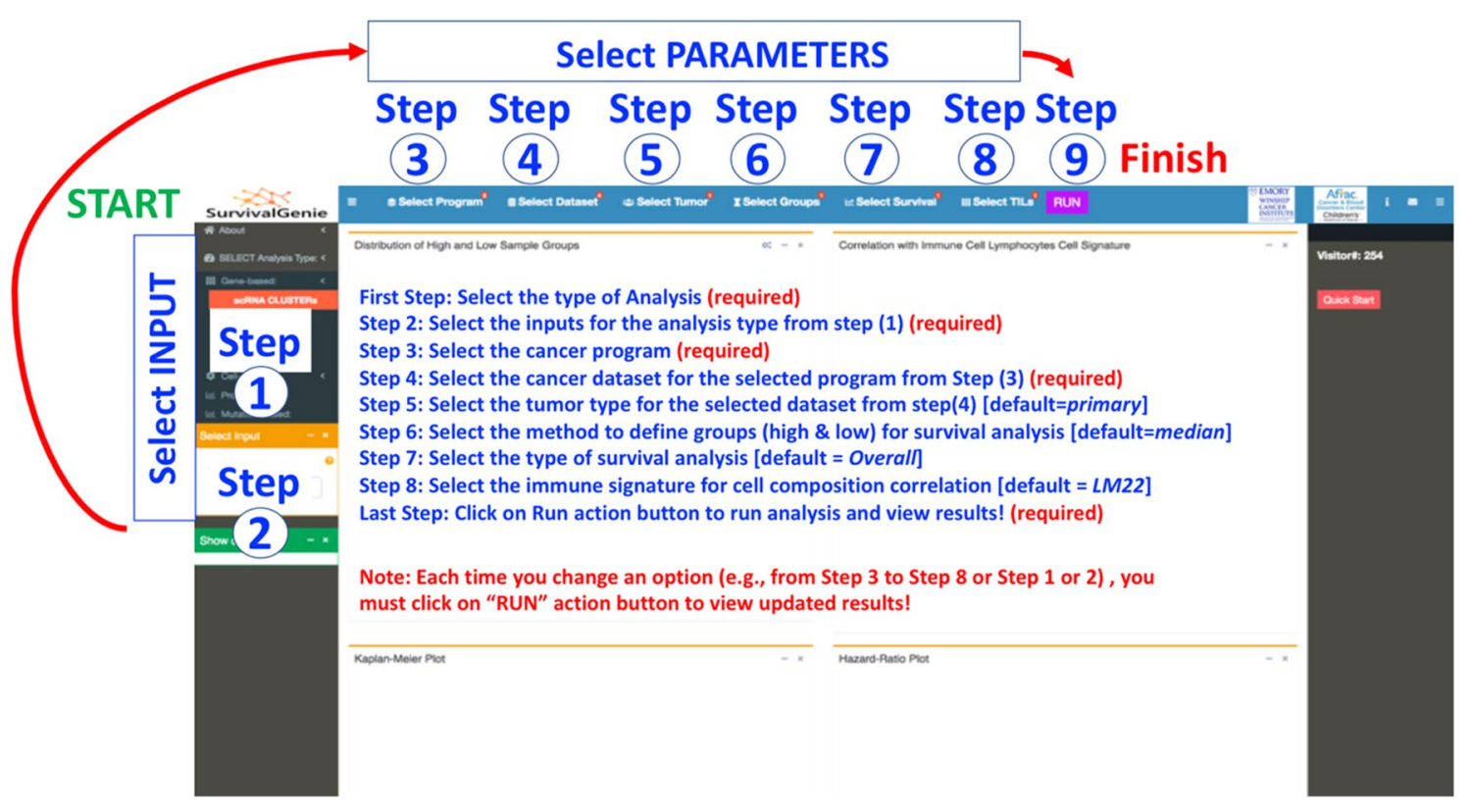

Figure 2. Summary of steps for performing survival analysis using the Survival Genie platform. Detailed instructions for analysis options and procedure are available in the demonstration tutorial video (https://www. youtube.com/watch?v=H5s6OYvwwoo) and the user manual on the GitHub site (https://github.com/bhasin-lab/ SurvivalGenie).

Single-cell RNA-seq clusters markers file containing lists of significant gene markers for each identified cell cluster derived from any single-cell RNA-seq experiment using analysis tools such as Seurat ${ }^{20}$. An adapted ssGSEA is implemented to compute enrichment scores (ES) for marker genes for identified clusters from the single-cell experiment. Each tumor cluster enrichment score is then divided into two risk categories to predict patient outcomes and associations to tumor cell composition. Additionally, the Genes Ratio input option allows users to perform survival analysis on the ratio of two genes computed from the normalized expression values, and lastly (iii) Single Gene input option allows users to perform single-gene queries to study associations with clinical out- 
comes. (b) Cell-based is to query cell types derived from (i) CIBERSOFT TILs proportion estimates of tumorinfiltrating lymphocytes (TILs) using LM6 and LM22 cell signature matrix ${ }^{21}$. The proportions were estimated using bulk tumor normalized FPKM expression data for each tumor for cancer types following the CIBERSORT method $^{21}$ and (ii) Digital TILs percentage retrieved based on H\&E images from The Cancer Imaging Archive ${ }^{14}$. (c) Profile-based input option is to query weighted gene profiles derived from weighted gene co-expression network analysis (e.g., 18-gene T-cell inflammation gene expression profile, the so-called Gene Expression Profile, GEP scores). The GEP score is calculated as the weighted sum of the normalized FPKM expression values of the gene signature for each tumor. The weightings for each HGNC approved gene symbol in the signature are provided by the user in the uploaded submission file. (d) Mutation-based input option is to query tumor mutation burden (TMB) within a cancer dataset based on all, only non-synonymous or exonic somatic mutations per megabase $(\mathrm{Mb})$ of the genome.

Cancer datasets, tumor types, optimal cut-offs, and survival options. In Survival Genie, users have options to select cancer program, tumor type, and methods for partitioning expression profiles (Steps 3-9, Fig. 2). The comprehensive analysis parameter includes (3) Selection of a cancer program from the available list that includes TCGA, TARGET, and MMRF CoMMpass. (4) Selection of cancer datasets from selected cancer programs to perform survival analysis. (5) Selection of the tumor type, primary, recurrent, or metastatic within the selected cancer dataset. The majority of cancer datasets consist of primary tumor samples with the exception of skin melanoma (TCGA-SKCM) that is dominated by metastatic tumor samples. (6) Selection of partitioning methods to define the dichotomous patient groups. There are four different partitioning methods (i.e., mean, median, percentile, and cutp) to subset the tumors into high and low groups to assess the association of expression levels with survival outcomes. The Percentile-based partitioning method allows the selection of desired upper and lower thresholds (e.g., quartiles or 10th vs. 90th percentile) to subset tumor samples into low and high groups. The optimal cut-point Cutp option estimates the martingale residuals ${ }^{6}$ using the 'survMisc ${ }^{22}$ package to divide patients into high and low groups. (7) Selection of survival analysis from either overall or event-free survival time, although the latter is only applicable to pediatric cancer datasets from the TARGET program. (8) Select TILs signature based on 22 (LM22) or 6 (LM6) immune cell types. The relative fractions of cell types are estimated for LM6 and LM22 immune cell gene signature from bulk tumors FPKM gene expression data using the CIBERSORT deconvolution method ${ }^{21}$. (9) Submit analysis to perform analysis after making appropriate selections of input parameters.

Analysis outputs and visualization. The results from the gene set and gene ratio-based analysis are displayed in five separate tabs: (A) Expression levels of high and low sample groups display either a box and whisker or bar plots showing the distribution of estimated molecular profile (e.g., gene expression, the expression ratio of two genes, gene set enrichment scores, cell proportion, or mutation burden) (Fig. 3A). (B) Correlation with lymphocytes cell signature shows the colored grid matrix of Pearson correlation of CIBERSOFT deconvoluted lymphocytes RNA-seq gene expression data and the estimated molecular data profile (Fig. 3B). Correlation coefficients are indicated with a color gradient, blue color represents negative correlation, while red color represents the positive correlation. The shape denotes the significance of the correlation with squares showing significant associations ( $p$-value $<0.05)$. (C) Kaplan-Meier $(\mathrm{KM})$ plot shows the KM survival curves in the stratified high (red) and low (blue) groups of patients with log-rank test (Fig. 3C). A log-rank $p$-value $<0.05$ is considered statistically significant. (D) Hazard ratio (HR) plot shows the survival significance by HR and HR $p$-value (Fig. 3D). $\mathrm{HR}$ is estimated based on a univariate Cox proportional hazard regression model with Wald test. An HR value above 1 indicates an increase in hazard (poor outcome) while an HR value below 1 indicates a reduction in the hazard (good outcome), and an HR value equal to 1 indicates no effect. A $p$-value $<0.05$ is considered statistically significant. (E) The forest plot shows the detailed table of the univariate Cox regression survival analysis (Fig. 3E). The plot shows the hazard ratio and 95\% confidence intervals associated with two groups considered in the univariable analysis along with Wald test and log-rank $p$-values. The table also shows the cut-off values applied to subset the patients into high and low groups along with the sample numbers in each group. The squares represent the HR, and the horizontal lines depict the upper and lower limits of the HR 95\% confidence interval. Significant associations are shown in red-filled HR value squares. An arrow at the end of the horizontal line indicates the upper limit of the $95 \%$ confidence interval is higher than the maximum shown (i.e., 3 ). Additionally, the "Show Output" option (Fig. 3) allows users to select and view the plots (A) and (C) based on input clusters (for scRNA cluster analysis) or datasets (for single-gene analysis).

Use case examples using Survival Genie. Use case 1: survival analysis of hallmark gene sets. We performed Cox proportional hazards regression analysis to examine the correlation between hallmark gene sets $(n=50)$ obtained from the Molecular Signatures Database (MSigDB) $)^{23,24}$ and survival (OS) across pediatric and adult cancers. Single sample enrichment scores were computed for each hallmark gene set across all cancer types using the ssGSEA method. The "survival" R package was utilized to calculate log-rank $p$-values, hazard ratios (HR), and 95\% confidence intervals (CI). Hierarchical clustering analysis (HCA) was performed on HR to determine survival patterns of hallmark gene sets $(n=50)$ and cancer type (Fig. 4, Supplementary Table 2).

The HCA on hallmark gene sets HR segregated cancers into three major groups (i.e., good, poor, and mixed outcomes) based on survival associations (Fig. 4). Interestingly, across all the hallmark gene sets, pediatric lymphoblastic leukemia (i.e., TARGET-ALL-P2) shows a consistent pattern of overall good survival whereas TCGA low-grade glioma (TCGA-LGG) showed overall bad survival. The association might be driven by overall low relapse rate for pediatric ALL and bad/short survival associated with low-grade glioma. The chronic inflammatory response-related gene sets such as "IL2 STAT5 signaling", "interferon-gamma response", "interferon-alpha 


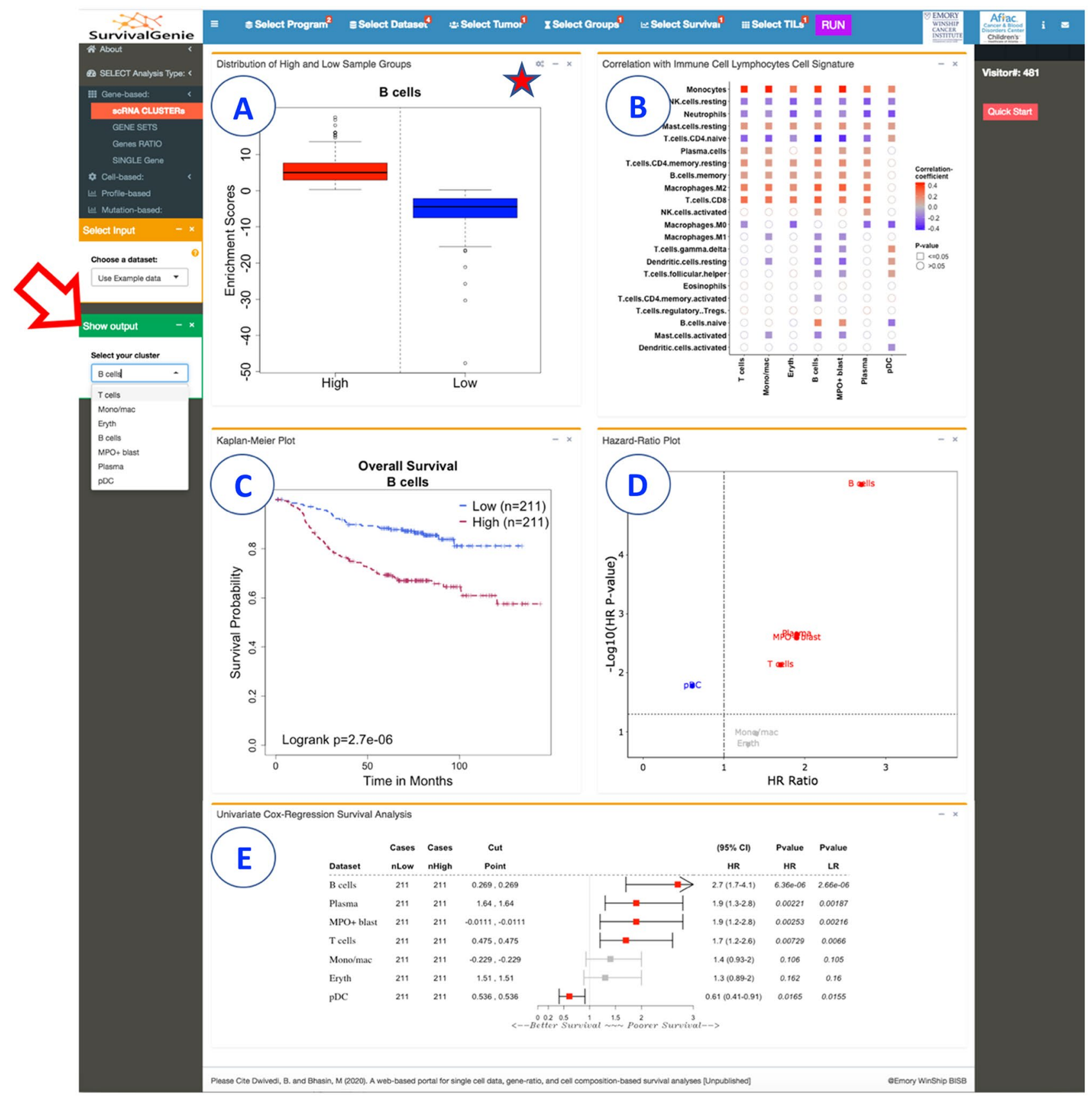

Figure 3. Summary of Survival Genie gene set analysis showing the five tabulated outputs: (A) distribution of gene expression from high and low sample groups, (B) correlation with immune cell signature, (C) KaplanMeier, (D) hazard ratio, and (E) forest plot.

response", "inflammatory response", "apoptosis", and "p53 pathway" were found to be significantly associated with poor overall survival across multiple adult cancers including TCGA-LGG, TCGA-UVM, and TCGA-PADD datasets.

Use case 2: survival association of genes from hallmark gene sets. We performed single-gene analysis to explore further whether gene set analysis outcomes are influenced at the individual gene level survival associations. Cox regression analysis was performed using the RNA-seq expression of genes $(n=4383)$ from 50 hallmark gene sets. The results of survival analysis across 47 types of cancer for individual genes from hallmark gene sets are listed in Supplemental Table 3. We computed the proportion of significant genes associated with patient survival from each hallmark gene set across tumor types (Fig. 5).

The top cancer types that contained the highest proportion of hallmark genes significantly associated with good overall survival are TARGET-ALL-P2, CPTAC-3, TCGA-KIRC (Fig. 5A). On the other hand, most of the hallmark genes depicted association with poor outcomes in TCGA-LGG, TCGA-LIHC, TCGA-UVM, 


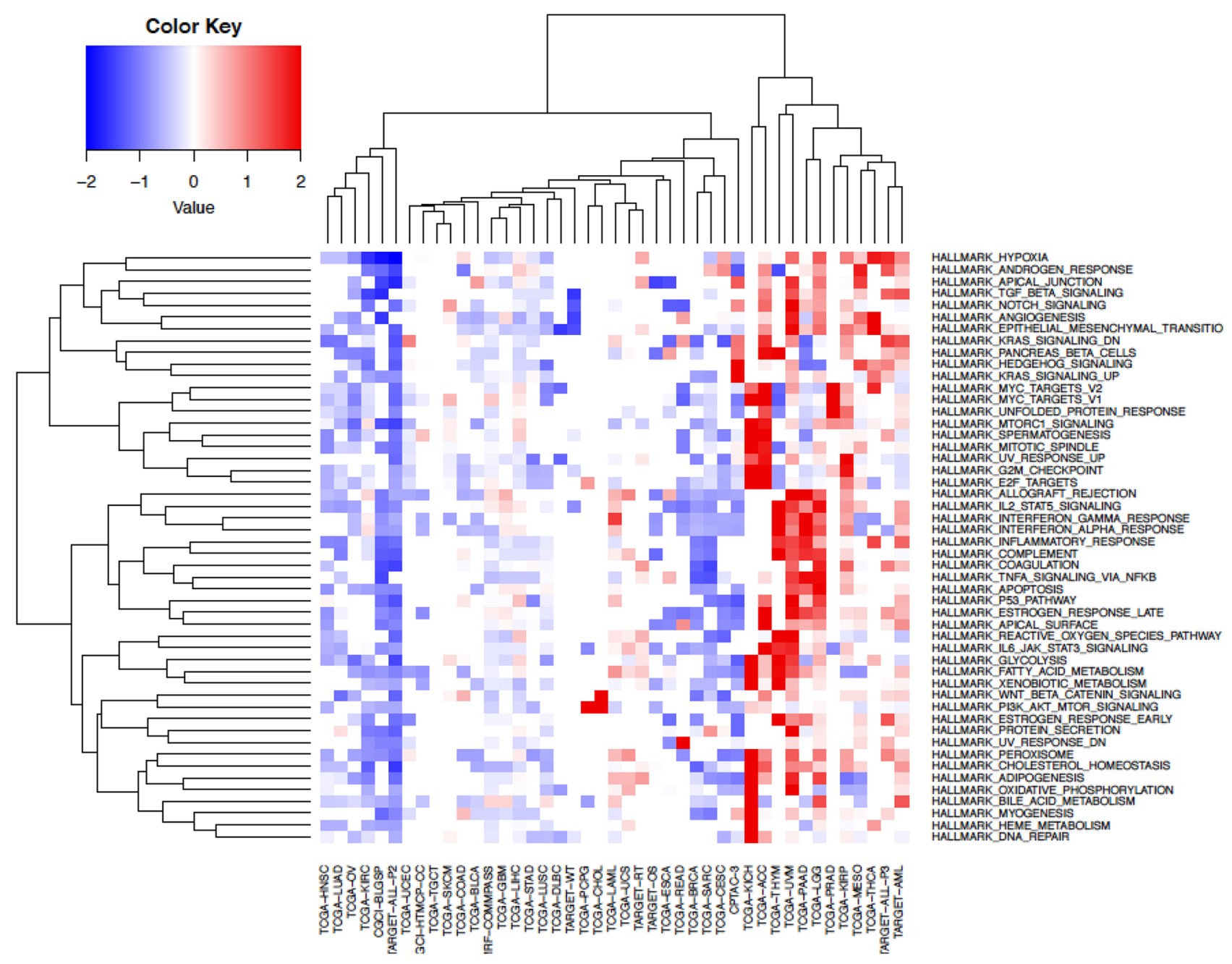

Figure 4. Gene sets-based survival analysis. Hierarchical clustering of Broad hallmark-associated gene sets and cancer types. The predicted survival outcomes are visualized using Euclidean distance and complete linkage method, using hazard ratios values for each hallmark associated gene set $(n=50)$ across all cancer datasets $(\mathrm{n}=43)$.

TCGA-ACC cancers (Fig. 5B). Regardless of the cancer types, genes such as ITGA5, SERPINE1, EIF4EBP1 are majorly associated with bad outcomes; while genes such as ALDH2, DBP, FRAT1, PDCD4 are associated with good outcomes.

Single-gene analysis of low-grade gliomas (TCGA-LGG) that showed poor prognosis at the gene set level depicted approximately $30-40 \%$ genes associated with poor outcomes (hazard ratio $>1$ and $p$-value $<0.05$ ) (Fig. 5B). These genes belong mainly to immune response and inflammatory gene sets such as HALLMARK inflammatory response, HALLMARK interferon-gamma response, HALLMARK TNFA signaling via NF-KB, and HALLMARK IL2 STAT5 signaling (Fig. 5B).

Similarly, single-gene analysis of TARGET pediatric acute lymphoid leukemia (TARGET-ALL-P2) showed that $40-50 \%$ from hallmark gene sets correlated with good outcomes (Fig. 5A). The majority of these genes belong to metabolic and inflammatory gene sets such as HALLMARK interferon-gamma response, HALLMARK glycolysis, HALLMARK KRAS signaling down, and HALLMARK adipogenesis.

Use case 3: survival analysis of T cell exhaustion to effector marker genes ratio. To determine the impact of the ratio of exhausted and functional $\mathrm{T}$ cells on cancer outcomes, we performed gene ratio-based analysis using the Survival Genie platform. For the analysis, we have used expression levels of Layilin (LAYN), a potent maker for quantifying tumor-infiltrating exhausted $\mathrm{CD} 8^{+} \mathrm{T}$ cells ${ }^{25}$. We have chosen GZMA and IFNG as markers for quantifying functional and activated $\mathrm{CD} 8^{+} \mathrm{T}$ cells ${ }^{26}$. We explored the effect of exhausted to effector $\mathrm{CD}^{+} \mathrm{T}$ cell ratio on survival outcome across adult and pediatric cancers.

The RNA-seq normalized expression values were used to compute the ratios of LAYN to GZMA and LAYN to IFNG for each tumor sample. The results of Cox regression analysis across 47 cancer types for each gene ratio, i.e., LAYN: GZMA and LAYN: IFNG are shown in Fig. 6. The higher LAYN: GZMA ratio (Fig. 6A) showed a significant association with poor survival in bladder, breast, head and neck, lung, liver, stomach, thyroid, and 

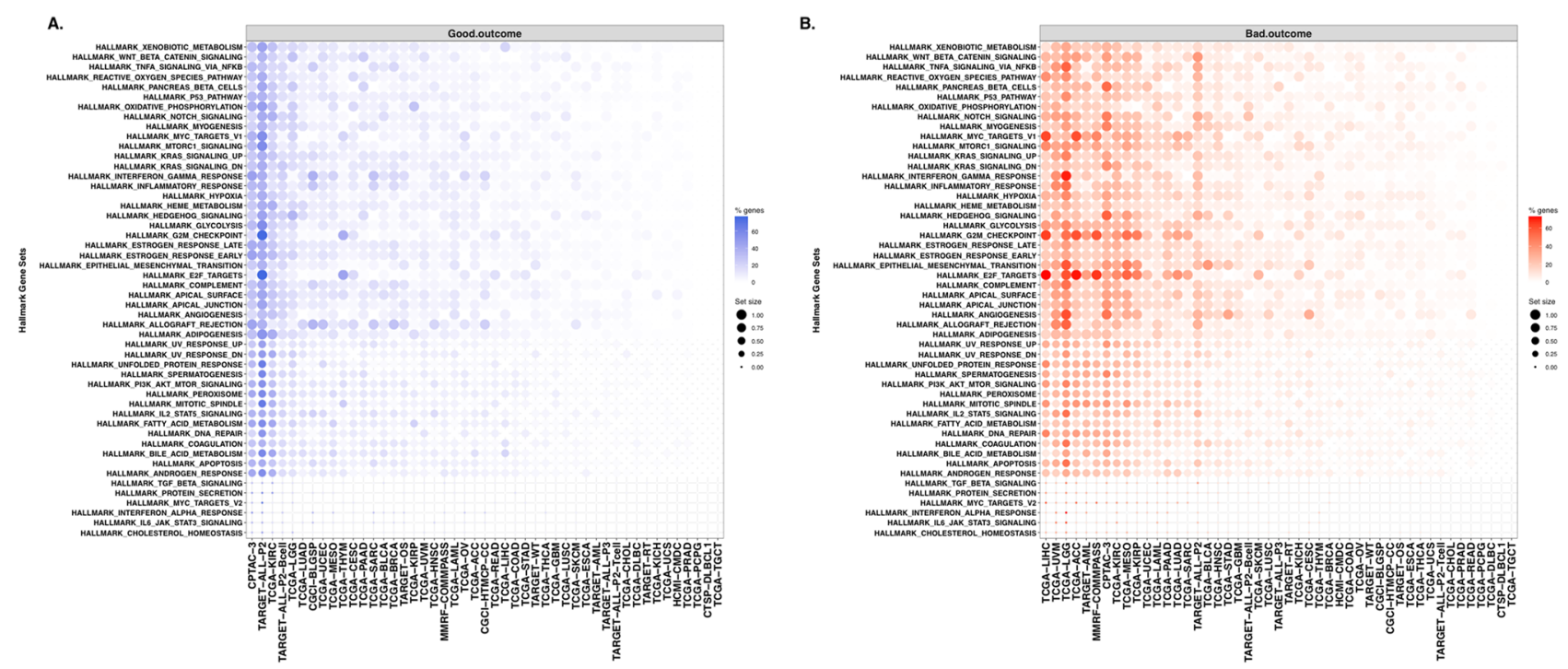

Figure 5. Hallmark genesets with enrichment of survival-associated genes. Summary of significantly associated hallmark genes with good (A) and bad (B) overall survival for each hallmark gene set for each cancer type. The dot color gradient represents the percentage of genes per gene set (\% genes) that are significantly associated ( $p$-value $<0.05)$ with survival outcome, while the size of the dots represents the total number of genes within a gene set (set size). The blue and orange color gradients indicate the good $(\mathrm{HR}<1)$ and bad $(\mathrm{HR}>1)$ survival outcomes.
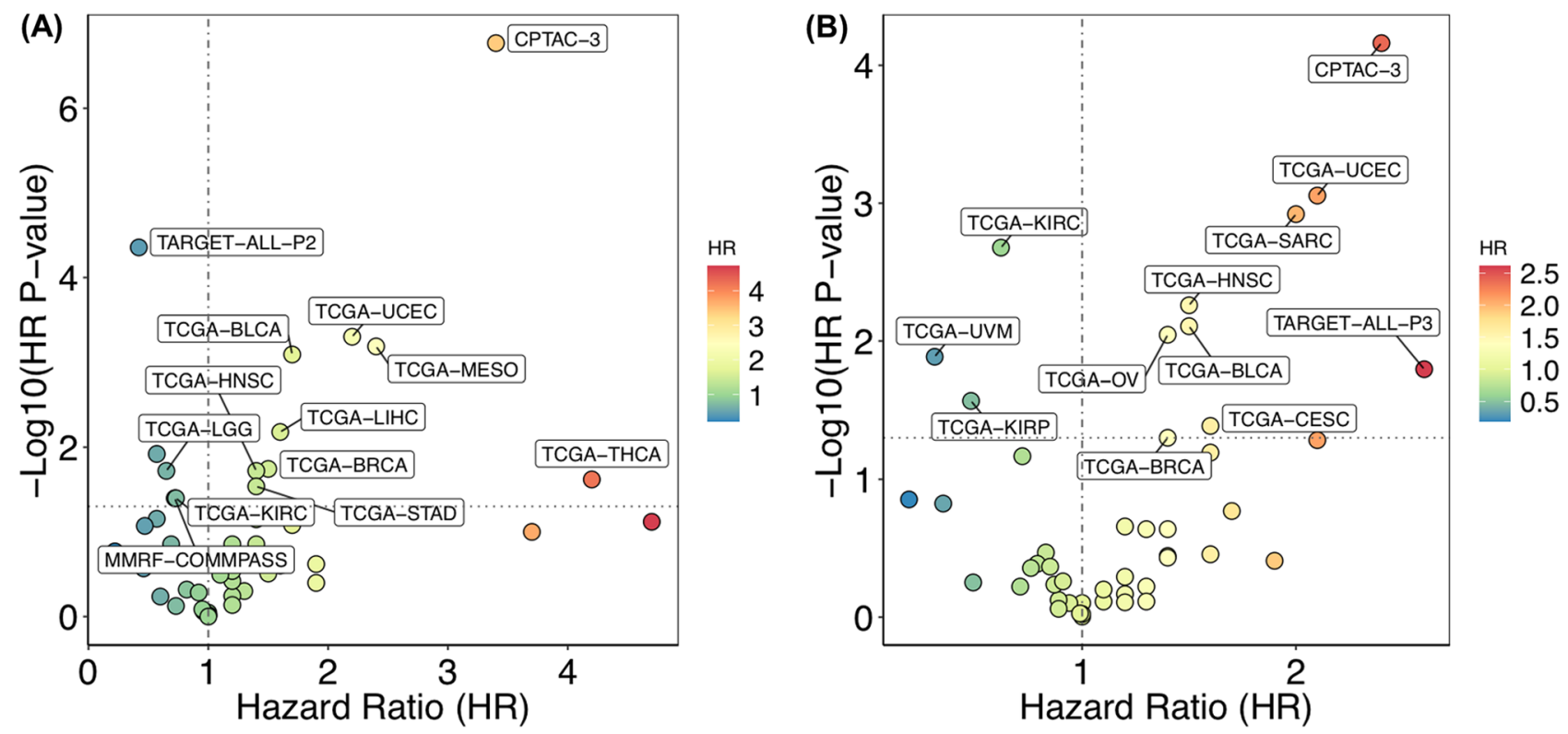

Figure 6. Gene ratio-based survival analysis. Association of (A) LAYN: GZMA and (B) LAYN: IFNG gene ratios on patient survival in different types of cancer. Only the significantly associated cancer types are labeled.

uterine cancer (Supplementary Table 4); while low LAYN: GZMA expression ratios were significantly associated with better prognosis in multiple myeloma, leukemia, low-grade glioma, and kidney cancer. A similar significant association of high LAYN to IFNG ratio with poor survival could also be observed in CPTAC-3, TCGA-BLCA, TCGA-BRCA, TCGA-HNSC, TCGA-UCEC (Fig. 6B). On the contrary, in datasets from TCGA-KIRC, TCGAKIRP, and TCGA-UVM higher ratios of LAYN: IFNG was associated with better survival. These data suggest that high expression of exhausted T cells marker (or lower expression of effector T cell marker) tend to be associated with a poor prognosis. However, high expression of cytolytic markers, GZMA or IFNG tend to suggest a good prognosis in blood and kidney cancers, respectively.

Use case 4: survival analysis of single-cell RNA-seq T cell clusters. We illustrate the utility of Survival Genie for scRNA-seq cluster markers prognostic utility by implementing it for survival analysis on $\mathrm{T}$ cell 

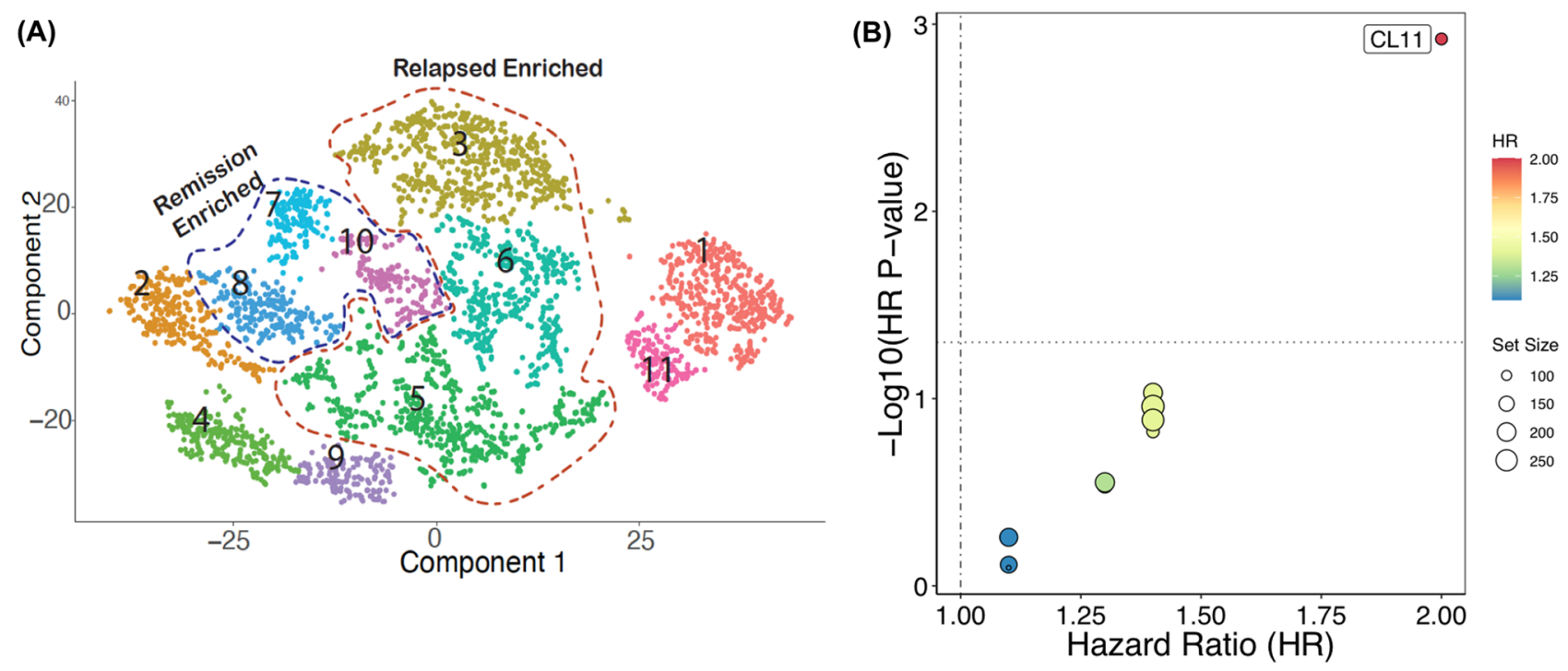

Figure 7. Single-cell clusters-based survival analysis. (A) UMAP of single-cell RNA-seq data from relapseassociated non-AML cells at the time of diagnosis. The colors represent the 11 distinct sub-clusters of T cells. (B) Prognostic significance of identified scRNA-seq clusters on overall survival in TCGA-LAML patient dataset. The significant associations between cluster enrichment and OS are highlighted.

clusters (Fig. 7A) generated from scRNA-seq analysis of paired pediatric AML bone marrow samples taken at the time of diagnosis and end of induction ${ }^{27}$. We performed Cox proportional survival analysis using TCGA-LAML dataset (Fig. 7). Out of the $11 \mathrm{~T}$ cell clusters, the relapse-associated cluster (\#CL11) depicted a significant association with poor survival $(\mathrm{HR}=2, p$-value $=0.001)$ (Fig. 7B). Moreover, cluster 11 (\#CL11) depicted overexpression of CD69, a type II glycoprotein that is known to regulate inflammation and exhaustion of tissue-resident $\mathrm{T}$ cells and promote tumor growth/relapse ${ }^{28}$.

\section{Discussion}

In this genomics era with abundant cancer genomics data, the bioinformatics tools to explore the prognostic utility of individual genes or gene sets are a major bottleneck in identifying robust prognostic biomarkers using the power of big data. Despite the multiple existing survival analysis tools, these tools have multiple shortcomings including data comprehensiveness and survival analysis on gene markers from single-cell data or cell fractions. Using traditional transcriptomic data, a plethora of individual genes have been tested and numerous suggested as potential prognosis markers for different cancer. As intuitively obvious to calculate single sample enrichment scores for a gene set per sample to test prognostic utility, it is not routinely used for survival analysis. With Survival Genie, researchers can easily explore multi-gene markers at once and identify gene sets associated with cancer survival. The analytical options in Survival Genie make it a unique resource for predicting clinical response based on profiles other than single-gene or average multiple-gene expression across different cancers with extended functionality to evaluate survival associations of single-cell clusters.

With multiple data sources and no consensus on cross-platform data processing and normalization, data standardization and optimization, cohort size, and analysis workflows, different survival tools yield different results. To maintain uniformity in the genomic data, while developing Survival Genie we have used harmonized cancer datasets from the NCI Genomic Data Commons. At present, GDC ${ }^{4}$ contains multidimensional datasets from 68 cancer programs, all processed and analyzed with the latest human reference genome build GRCh38 and up-to-date workflows. This uniformly processed data allows comparative analysis of prognostic genes or genesets with minimal bias resulting from inconsistent data preprocessing, normalization, or technological differences among datasets. To further enhance the comprehensiveness of Survival Genie, we are planning to include data from other cancer genomics initiatives including cancer projects such as BEATAML1.0 ${ }^{29}$, Cancer Genome Characterization Initiative (CGCI), and Cancer Dream Team projects.

Exploring the prognostic utility of different malignant and immune cells clusters from single-cell data allows exploration of prognostic utility of heterogeneous malignant tumor cells along with the immune microenvironment. With bulk RNA-seq data, we may not be able to determine how single-cell signatures are related to cell-level composition during tumor progression. This certainly holds true when studying rare subclonal profiles in cancers such as acute myeloid leukemia. To address this limitation, we estimated the cellular composition of tumors from bulk RNA-seq expression by deconvolution, using immune cell-type-specific profiles from tumor-derived single-cell RNA sequencing data ${ }^{21}$. The resulting cell correlation matrix can subsequently be used to identify the cell type abundance in bulk tissue data when predicting outcomes.

We have improved upon the existing tools in the ability to predict prognosis from multi-gene marker sets. In the future, efforts will be made to include options for multivariable regression and in-depth subtype analysis using clinicopathological features (tumor grade, lymph node status, treatment, mutation status, subtypes, tumor tissue image, microsatellite instability). In addition, Survival Genie will also allow exploration of prognostic associations 
of miRNA, long non-coding RNA, and hyper-/hypomethylated genomic regions. We also intend to include the ability to subset survival analysis based on genes that correlate with canonical cell-specific markers (e.g., T cells), thus allowing survival analysis based on cell-specific signatures. Running and being tested since September 2020, Survival Genie is an open-source web-based tool we intend to maintain it for a minimum of five years.

\section{Conclusion}

In summary, Survival Genie allows the exploration of the prognostic potential of genes and gene sets in a broad range of cancer datasets and specifically to validate the cell-type-specific markers from single-cell mRNA sequencing. The exploration of the three-way relationship between the cell-specific biomarker genes, enrichment of cell types and survival outcomes will assist in understanding how specific genes impact the cellular composition of the tumor microenvironment to drive cancer outcomes. This will assist in developing biomarkers to predict the immune-suppressive microenvironment that might lead to the identification of next-generation candidates for targeted immunotherapies.

Received: 29 September 2021; Accepted: 3 February 2022

Published online: 23 February 2022

\section{References}

1. Cancer Genome Atlas Research, N et al. The Cancer Genome Atlas Pan-Cancer analysis project. Nat. Genet. 45, 1113-1120. https:// doi.org/10.1038/ng.2764 (2013).

2. International Cancer Genome, C et al. International network of cancer genome projects. Nature 464, 993-998. https://doi.org/10. 1038/nature08987 (2010).

3. Ma, X. et al. Pan-cancer genome and transcriptome analyses of 1,699 paediatric leukaemias and solid tumours. Nature 555, 371-376. https://doi.org/10.1038/nature25795 (2018).

4. Grossman, R. L. et al. Toward a shared vision for cancer genomic data. N. Engl. J. Med. 375, 1109-1112. https://doi.org/10.1056/ NEJMp1607591 (2016).

5. Zheng, H. et al. Comprehensive review of web servers and bioinformatics tools for cancer prognosis analysis. Front. Oncol. 10, 68. https://doi.org/10.3389/fonc.2020.00068 (2020).

6. Cerami, E. et al. The cBio cancer genomics portal: An open platform for exploring multidimensional cancer genomics data. Cancer Discov. 2, 401-404. https://doi.org/10.1158/2159-8290.CD-12-0095 (2012).

7. Chandrashekar, D. S. et al. UALCAN: A portal for facilitating tumor subgroup gene expression and survival analyses. Neoplasia 19, 649-658. https://doi.org/10.1016/j.neo.2017.05.002 (2017).

8. Borcherding, N., Bormann, N. L., Voigt, A. P. \& Zhang, W. TRGAted: A web tool for survival analysis using protein data in the Cancer Genome Atlas. F1000Research 7, 1235. https://doi.org/10.12688/f1000research.15789.2 (2018).

9. Györffy, B. Survival analysis across the entire transcriptome identifies biomarkers with the highest prognostic power in breast cancer. Comput. Struct. Biotechnol. J. 19, 4101-4109 (2021).

10. Gyorffy, B. et al. An online survival analysis tool to rapidly assess the effect of 22,277 genes on breast cancer prognosis using microarray data of 1,809 patients. Breast Cancer Res. Treat. 123, 725-731. https://doi.org/10.1007/s10549-009-0674-9 (2010).

11. Wang, F. et al. OSuvm: An interactive online consensus survival tool for uveal melanoma prognosis analysis. Mol. Carcinog. 59, 56-61. https://doi.org/10.1002/mc.23128 (2020).

12. Pak, K. et al. A user-friendly, web-based integrative tool (ESurv) for survival analysis: Development and validation study. J. Med. Internet Res. 22, e16084. https://doi.org/10.2196/16084 (2020).

13. GenomicDataCommons: NIH/NCI Genomic Data Commons Access (2021).

14. Clark, K. et al. The Cancer Imaging Archive (TCIA): Maintaining and operating a public information repository. J. Digit. Imaging 26, 1045-1057. https://doi.org/10.1007/s10278-013-9622-7 (2013).

15. A Package for Survival Analysis in R (2021).

16. Bioconductor Open Source Software for Bioinformatics v. 3.13.

17. R: A language and environment for statistical computing. (R Foundation for Statistical Computing, 2017).

18. Shiny R web-server.

19. Barbie, D. A. et al. Systematic RNA interference reveals that oncogenic KRAS-driven cancers require TBK1. Nature 462, 108-112. https://doi.org/10.1038/nature08460 (2009).

20. Hao, Y. et al. Integrated analysis of multimodal single-cell data. Cell 184, 3573-3587.e3529. https://doi.org/10.1016/j.cell.2021.04. $048(2021)$.

21. Chen, B., Khodadoust, M. S., Liu, C. L., Newman, A. M. \& Alizadeh, A. A. Profiling tumor infiltrating immune cells with CIBERSORT. Methods Mol. Biol. 1711, 243-259. https://doi.org/10.1007/978-1-4939-7493-1_12 (2018).

22. survMisc: Miscellaneous Functions for Survival Data (2018).

23. Liberzon, A. et al. The Molecular Signatures Database (MSigDB) hallmark gene set collection. Cell Syst. 1, 417-425. https://doi. org/10.1016/j.cels.2015.12.004 (2015).

24. Liberzon, A. et al. Molecular signatures database (MSigDB) 3.0. Bioinformatics 27, 1739-1740. https://doi.org/10.1093/bioinforma tics/btr260 (2011).

25. Zheng, C. et al. Landscape of infiltrating T cells in liver cancer revealed by single-cell sequencing. Cell 169, 1342-1356.e1316. https://doi.org/10.1016/j.cell.2017.05.035 (2017).

26. Roufas, C. et al. The expression and prognostic impact of immune cytolytic activity-related markers in human malignancies: A comprehensive meta-analysis. Front. Oncol. 8, 27. https://doi.org/10.3389/fonc.2018.00027 (2018).

27. Thomas, B. E. et al. Single cell transcriptomics revealed AML and non-AML cell clusters relevant to relapse and remission in pediatric AML. Blood 136(Supplement 1), 24-25. https://doi.org/10.1182/blood-2020-142513 (2020).

28. Mita, Y. et al. Crucial role of CD69 in anti-tumor immunity through regulating the exhaustion of tumor-infiltrating T cells. Int. Immunol. 30, 559-567. https://doi.org/10.1093/intimm/dxy050 (2018).

29. Tyner, J. W. et al. Functional genomic landscape of acute myeloid leukaemia. Nature 562, 526-531. https://doi.org/10.1038/s41586018-0623-z (2018).

\section{Acknowledgements}

We would like to thank the Winship Research Informatics shared resource of the Winship Cancer Institute of Emory University for supporting the web server. Thanks to Beena E. Thomas, Jonathan C. Patton, and Gulay B. Ulukaya for reviewing and editing the manuscript. 


\section{Author contributions}

B.D.: collected data, performed analysis, prepared manuscript. H.M., S.S.: prepared webtool and GitHub. S.B.: prepared webtool and GitHub, performed analysis, prepared manuscript. M.B.: supervised project, the designed analytical plan, wrote manuscript.

\section{Funding}

Research reported in this publication was supported in part by the Bioinformatics and Systems Biology Shared Resource of Winship Cancer Institute of Emory University and NIH/NCI under award number P30CA138292. Research is also supported through Emory University startup funding for Dr. Bhasin. The research is also supported by Precision Medicine funding by CURE foundation. The content is solely the responsibility of the authors and does not necessarily represent the official views of the National Institutes of Health.

\section{Competing interests}

The authors declare no competing interests.

\section{Additional information}

Supplementary Information The online version contains supplementary material available at https://doi.org/ 10.1038/s41598-022-06841-0.

Correspondence and requests for materials should be addressed to M.B.

Reprints and permissions information is available at www.nature.com/reprints.

Publisher's note Springer Nature remains neutral with regard to jurisdictional claims in published maps and institutional affiliations.

(c) (i) Open Access This article is licensed under a Creative Commons Attribution 4.0 International License, which permits use, sharing, adaptation, distribution and reproduction in any medium or format, as long as you give appropriate credit to the original author(s) and the source, provide a link to the Creative Commons licence, and indicate if changes were made. The images or other third party material in this article are included in the article's Creative Commons licence, unless indicated otherwise in a credit line to the material. If material is not included in the article's Creative Commons licence and your intended use is not permitted by statutory regulation or exceeds the permitted use, you will need to obtain permission directly from the copyright holder. To view a copy of this licence, visit http://creativecommons.org/licenses/by/4.0/.

(C) The Author(s) 2022 